\title{
Malignant Extracranial Germ Cell Tumours: A First Report by the South African Children's Cancer Study Group
}

Marc Hendricks ${ }^{1}$, Annibale Cois ${ }^{2}$, Jennifer Geel ${ }^{3}$, Jan du Plessis ${ }^{4}$, Mairi Bassingthwaighte ${ }^{5}$, Gita Naidu ${ }^{6}$, Biance Rowe ${ }^{7}$, Ane Buchner ${ }^{8}$, Fareed Omar ${ }^{9}$, Karla Thomas ${ }^{10}$, Ronelle Uys ${ }^{11}$, Anel van Zyl ${ }^{11}$, Jaques van Heerden ${ }^{12}$, Ngoakoana Mahlachana ${ }^{3}$, Johani Vermeulen ${ }^{13}$, Alan Davidson $^{14}$, Lindsay Frazier ${ }^{15}$, Kirsty Donald ${ }^{16}$, and Mariana Kruger ${ }^{17}$

${ }^{1}$ University of Cape Town

${ }^{2}$ Stellenbosch University

${ }^{3}$ University of the Witwatersrand

${ }^{4}$ University of the Free State Faculty of Health Sciences

${ }^{5}$ Division of Paediatric Haematology Oncology, Chris Hani Baragwanath Academic

Hospital, University of Witwatersrand, Soweto, South Africa

${ }^{6}$ Chris Hani Baragwanath Hospital

${ }^{7}$ Division of Paediatric Haematology Oncology, Chris Hani Baragwanath Academic

Hospital, University of Witwatersrand, Soweto, South Africa.

${ }^{8}$ University of Pretoria

${ }^{9}$ Steve Biko Academic Hospital and the University of Pretoria

${ }^{10}$ Paediatric Haematology Oncology, Department of Paediatrics and Child Health, Frere

Hospital, East

${ }^{11}$ Stellenbosch University Faculty of Medicine and Health Sciences

${ }^{12}$ Paediatric Haematology and Oncology, Department of Paediatrics and Child Health, University of Antwerp, Antwerp University Hospital, Antwerp, Belgium

${ }^{13}$ Paediatric Haematology Oncology, Department of Paediatrics and Child Health, Port Elizabeth Provincial Hospital, Walter Sisulu University, Port Elizabeth, South Africa

${ }^{14}$ Red Cross War Memorial Children's Hospital and the University of Cape Town

${ }^{15}$ Dana Farber Cancer Institute

${ }^{16}$ Division of Developmental Paediatrics, Department of Paediatrics and Child Health, Red Cross War Memorial Children's Hospital, and the Neuroscience Institute, University of Cape Town, Cape Town, South Africa.

${ }^{17}$ Tygerberg Hospital and Stellenbosch University

December 6, 2021

\begin{abstract}
OBJECTIVE To determine the overall survival (OS) and prognostic factors influencing outcomes in children and adolescents with malignant extracranial germ cell tumours (MEGCTs) in preparation for the development of a harmonised national treatment protocol.METHODS A retrospective folder review was undertaken at nine South African paediatric oncology units to document patient profiles, tumour and treatment-related data and outcomes for all children with biopsy proven MEGCTs from birth up to and including 16 years of age. RESULTS Between 1 January 2000 and 31 December 2015, 218 patients were
\end{abstract}


diagnosed with MEGCTs. Female sex (HR $0.284 \mathrm{p}=0.037)$ and higher socio-economic status (SES) (HR 0.071; p=0.039) were associated with a significantly lower risk of death. Advanced clinical stage at diagnosis significantly affected 5-year OS: stage I $-96 \%$; stage II - 94.3\%; stage III -75.5\%; ( $\mathrm{p}=0.017)$ and stage IV $(60.1 \%$; $<<0.001)$. There was a significant association between earlier stage at presentation and higher SES $(\mathrm{p}=0.03)$. Patients with a serum AFP level of more than 33,000 ng/ml at diagnosis had significantly poorer outcomes $(\mathrm{p}=0.002)$. The use of chemotherapy significantly improved survival, irrespective of the regimen used $(\mathrm{p}<0.001)$. CONCLUSIONS The cohort demonstrated a 5-year OS of $80.3 \%$ with an EFS of $75.3 \%$. Stage, the use of chemotherapy and an elevated serum AFP level of more than 33,000ng/ml were independently predictive of outcome. The relationship between SES and outcome is important as the implementation of the new national protocol hopes to standardise care across the socio-economic divide.

\section{Malignant Extracranial Germ Cell Tumours: A First National Report by the South African Children's Cancer Study Group}

Marc Hendricks, CMOPaed(SA) ${ }^{1}$, Annibale Cois, $\mathrm{PhD}^{2,13}$, Jennifer Geel, CMOPaed(SA) ${ }^{3}$, Johan du Plessis, $\mathrm{MPhil}^{4}$, Mairi Bassingthwaighte, CMOPaed(SA) ${ }^{5}$, Gita Naidu, $\mathrm{PhD}^{5}$, Biance Rowe, CMOPaed(SA) ${ }^{5}$, Ané Büchner, MPhil ${ }^{6}$, Fareed Omar, CMOPaed $(\mathrm{SA})^{6}$, Karla Thomas, CMOPaed(SA) ${ }^{7}$, Ronelle Uys, MBChB ${ }^{8}$, Anel van Zyl, CMOPaed(SA) ${ }^{8}$, Jaques van Heerden, $\mathrm{PhD}^{8,12}$, Ngoakoana Mahlachana, CMOPaed(SA) ${ }^{5}$, Johani Vermeulen, CMOPaed(SA) ${ }^{9}$, Alan Davidson, MPhil ${ }^{1}$, A. Lindsay Frazier L, MD ${ }^{10}$, Kirsty Donald, $\mathrm{PhD}^{11}$, Mariana Kruger $\mathrm{M}, \mathrm{PhD}^{8}$.

${ }^{1}$ Haematology Oncology Service, Department of Paediatrics and Child Health, Red Cross War Memorial Children's Hospital, University of Cape Town, Cape Town, South Africa.

${ }^{2}$ Division of Health Systems and Public Health, Department of Global Health, Stellenbosch University, Cape Town, South Africa.

${ }^{3}$ Division of Paediatric Haematology Oncology, Department of Paediatrics and Child Health, Charlotte Maxeke Johannesburg Academic Hospital, University of Witwatersrand, Johannesburg, South Africa.

${ }^{4}$ Division of Paediatric Haematology Oncology, Department of Paediatrics, Universitas Hospital, University of the Free State, Bloemfontein, South Africa.

${ }^{5}$ Division of Paediatric Haematology Oncology, Chris Hani Baragwanath Academic Hospital, University of Witwatersrand, Soweto, South Africa.

${ }^{6}$ Paediatric Haematology Oncology, Department of Paediatrics, Steve Biko Academic Hospital, University of Pretoria, Tshwane, South Africa.

7 Paediatric Haematology Oncology, Department of Paediatrics and Child Health, Frere Hospital, East London, South Africa.

${ }^{8}$ Paediatric Haematology Oncology, Department of Paediatrics and Child Health, Faculty of Medicine and Health Sciences, University of Stellenbosch, Tygerberg Hospital, Cape Town, South Africa.

${ }^{9}$ Paediatric Haematology Oncology, Department of Paediatrics and Child Health, Port Elizabeth Provincial Hospital, Walter Sisulu University, Port Elizabeth, South Africa.

${ }^{10}$ Paediatric Oncology, Dana Farber Cancer Institute / Boston Children's Cancer and Blood Disorder Centre, Harvard University, Boston, USA.

${ }^{11}$ Division of Developmental Paediatrics, Department of Paediatrics and Child Health, Red Cross War Memorial Children's Hospital, and the Neuroscience Institute, University of Cape Town, Cape Town, South Africa.

${ }^{12}$ Paediatric Haematology and Oncology, Department of Paediatrics and Child Health, University of Antwerp, Antwerp University Hospital, Antwerp, Belgium. 
${ }^{13}$ Division of Epidemiology and Biostatistics, School of Public Health and Family Medicine, University of Cape Town, Cape Town, South Africa.

*Correspondence to:

Marc Hendricks, MBChB, FCPaed(SA), CMOPaed(SA). Room 48, G1 OPD, Haematology Oncology Service, Red Cross War Memorial Children's Hospital, Klipfontein Road, Rondebosch, South Africa, 7700. Tel: +27 82870 2260, Email: Marc.Hendricks@uct.ac.za

Text word count 4205;

Abstract word count 244;

Brief running title: Paediatric MEGCTs in South Africa: A First National Report

Key words: Paediatric malignant extracranial germ cell tumours

Tables: 6

Figures: 6

Supplemental tables:1

Supplemental figures:1

Abbreviations

\begin{tabular}{ll}
\hline AFP & Alpha-fetoprotein \\
AIHA & Auto-immune haemolytic anaemia \\
AML & Acute myeloid leukaemia \\
BEP & Bleomycin, etoposide, cisplatin \\
B-HCG & Beta-choriogonadotropin \\
CI & Confidence interval \\
CINV & Chemotherapy induced nausea and vomiting \\
CRR & Complete resection rate \\
CT & Computed tomography \\
CXR & Plain film chest radiography \\
EFS & Event free survival \\
FIGO & International Federation of Obstetrics and Gynaecology \\
FN & Febrile neutropaenia \\
GFR & Glomerular filtration rate \\
ITs & Immature teratomas \\
ITP & Immune thrombocytopaenia \\
JEb & Carboplatin, etoposide, bleomycin (paediatric) \\
JGCTs & Juvenile granulosa cell tumours \\
LDH & Lactate dehydrogenase \\
LMICs & Low- and middle-income countries \\
MEGCTs & Malignant extra-cranial germ cell tumours \\
MRI & Magnetic resonance imaging \\
MTs & Mature teratomas \\
OS & Overall survival \\
PEb & Cisplatin, etoposide, bleomycin (paediatric) \\
PET & Positron emission tomography \\
POUs & Paediatric oncology units \\
SACCSG & South African Children's Cancer Study Group \\
SD & Standard deviation \\
SES & Socio-economic status
\end{tabular}


SLCTs Sertoli-Leydig cell tumours

UTI Urinary tract infections

WHO World Health Organisation

SIOP International, Lyon, France (Poster):

Hendricks M, Cois A, du Plessis J, Geel J, van Heerden J, Naidu G, Bassingwaighte M, Rowe, B, van Zyl A, Uys R, Büchner A, Omar F, Thomas K, Machlachana N, Vermeulen J, Davidson A, Donald K, Kruger M. Favourable outcomes for children with biopsy proven malignant extracranial germ cell tumours 1990-2015: A first national report by the South African Children's Cancer Study Group (SACCSG). Pediatr Blood Cancer , 2019, 66(11), SIOP 19-0822.

\section{ABSTRACT \\ OBJECTIVE}

To determine the overall survival (OS) and prognostic factors influencing outcomes in children and adolescents with malignant extracranial germ cell tumours (MEGCTs) in preparation for the development of a harmonised national treatment protocol.

\section{METHODS}

A retrospective folder review was undertaken at nine South African paediatric oncology units to document patient profiles, tumour and treatment-related data and outcomes for all children with biopsy proven MEGCTs from birth up to and including 16 years of age.

\section{RESULTS}

Between 1 January 2000 and 31 December 2015, 218 patients were diagnosed with MEGCTs. Female sex (HR $0.284 \mathrm{p}=0.037$ ) and higher socio-economic status (SES) (HR 0.071; $\mathrm{p}=0.039)$ were associated with a significantly lower risk of death. Advanced clinical stage at diagnosis significantly affected 5-year OS: stage I -96\%; stage II - 94.3\%; stage III -75.5\%; $(\mathrm{p}=0.017)$ and stage IV $(60.1 \%$; $<<0.001)$. There was a significant association between earlier stage at presentation and higher SES ( $\mathrm{p}=0.03)$. Patients with a serum AFP level of more than $33,000 \mathrm{ng} / \mathrm{ml}$ at diagnosis had significantly poorer outcomes $(\mathrm{p}=0.002)$. The use of chemotherapy significantly improved survival, irrespective of the regimen used $(\mathrm{p}<0.001)$.

\section{CONCLUSIONS}

The cohort demonstrated a 5 -year OS of $80.3 \%$ with an EFS of $75.3 \%$. Stage, the use of chemotherapy and an elevated serum AFP level of more than 33,000ng $/ \mathrm{ml}$ were independently predictive of outcome. The relationship between SES and outcome is important as the implementation of the new national protocol hopes to standardise care across the socio-economic divide.

\section{INTRODUCTION}

Malignant extracranial germ cell tumours (MEGCTs) represent a biologically heterogeneous group of malignancies with manifestation influenced by age, sex, site and the relationship of the tumour to puberty, although histologically they may be similar between sites $(1,2)$. MEGCTs are rare and comprise approximately $3 \%$ of all childhood tumours (3). However the incidence rises sharply at the time of puberty, and MEGCTs are the most common solid tumour in adolescents and young adults exhibiting similar behaviour (4). Traditional treatments included mainly surgery and chemotherapy, although testicular and mediastinal seminomas (5)(which are rare in children) and ovarian dysgerminomas are radiotherapy sensitive. The improved efficacy of chemotherapy regimens over time eclipsed the need for radiotherapy where undue toxicity could be avoided or minimised with chemotherapy alone. Consequently, radiotherapy has not been incorporated into first line treatment regimens in children. Instead radiotherapy continues to be used for refractory or relapsed tumours as a salvage strategy for poor chemotherapy responders and even then, has not been shown to add significant benefit (6). 
Einhorn and Donohue pioneered the first multi-agent chemotherapy regimens in adults with metastatic testicular disease in the late 1970s, initially using cisplatin, vinblastine and bleomycin which demonstrated impressive complete remission rates ( $80 \%$ and $74 \%$, respectively) $(7,8)$. Since that time several co-operative groups have used similar platinum backbones to refine chemotherapy approaches first in adults and then in paediatrics (9-12). Over time renal and ototoxicity prompted a switch from cisplatin to carboplatin, with etoposide and bleomycin (JEB) (10) which had been shown to be highly efficacious and less toxic by earlier investigators $(11,12)$. Subsequently JEb and PEb have been shown to have equivalence in the treatment of standard risk MEGCTs in children (13).

There is less reported data reported from low- and middle-income countries (LMICs), where resources are constrained and reliable paediatric cancer registries are confined to single institutions or do not exist at all (14). Recent data from the South African Children's Tumour Registry reported an age standardised ratio (ASR) of 1.7/1,000,000 for MEGCTs (15). Where data from LMICs is available, studies have shown comparable outcomes to high income countries (HICs). In South Africa these reports are limited to single institutions and have been shown to be setting appropriate, cost effective and efficacious (16). No published series exists that has investigated potential prognostic factors and treatment outcomes for MEGCTs in African paediatric populations, in particular the relationship between poverty and cancer outcomes. Rather, risk factors have been extrapolated from pooled data from much larger international collaborative groups in HICs $(17,18)$. As a preamble to the development of a national treatment protocol, the South African Children's Cancer Study Group (SACCSG) undertook a national retrospective review of all children 16 years and under with biopsy-proven MEGCTs.

\section{METHODS}

\section{Patients}

Nine paediatric oncology units across South Africa participated in the national retrospective study from the public and private sector. Anonymised demographic and treatment related data was collected at each site for all children with biopsy proven disease up to and including the age of 16 years of age. and included age, sex, anthropometry, symptom profiles, co-morbidities, stage, tumour site, histology, tumours markers, imaging techniques, response assessments at first review, timing and extent of tumour resections, chemotherapy regimens (neo-adjuvant and adjuvant), number of courses of chemotherapy (both first line and subsequent regimens), radiotherapy use, GFR and audiology monitoring, treatment sequelae and interventions, socioeconomic status by annual household income, nature of follow-up and outcome. Nutritional parameters were defined according to the WHO Nutritional Landscape Information System (NLIS) Interpretation Guide, 2010 (19), where moderate impact in each category was defined as an anthropometric measurement more than two standard deviations (SD) below the WHO Child Growth standards median and where severe impact was defined as more than three SD below that median. Socio-economic status was assigned according to the Provincial Government of the Western Cape's Federal Acquisition Regulation No.9 of 2017 (PGWC FAR9/2017). Tumours were staged according to the Children's Oncology Group staging system for extracranial germ cell tumours (2). This information was centralised into a single dataset exported in comma separated values format (.csv). Median follow-up was defined as the number of days from diagnosis to death or date last seen.

\section{Statistical Analysis}

Stata $^{T M}$ Statistical Software v. 14 (StataCorp, College Station, Texas) was used for consolidation, uniform recording and basic descriptive statistics. Survival analyses and output graphics were conducted with $R$ Statistical Environment v. 3.5.2 (R Core Team, Vienna, Austria). The Kaplan-Meier procedure was used to estimate overall survival (OS) curves and survival at specific time points (1, 2 and 5 years) with associated $95 \%$ confidence intervals $(20,21)$. The log-rank test was applied to assess the existence of significant differences between survival curves in different subgroups. Multivariate Cox regression models were fitted to analyse the association of the OS and the event-free survival (EFS) with a series of potential prognostic factors (22). Non-linear effects were analysed by introducing the relevant predictors as penalised smoothing 
splines (23). This method was employed to assess whether there may be an age threshold beyond which patients had a less favourable outcome and also to establish a similar threshold for serum AFP level at diagnosis, which influenced OS or EFS. The Spearman test was used to establish an association between ordinal variables, in this case SES and stage. The proportional hazards assumption was tested by computing the scaled Schoenfeld Residuals test (24). A p-value $<5 \%$ was used as a cut-off to define statistical significance. P-values for relative hazards were calculated in a post-hoc analysis comparing patients with AFP levels above and below the identified thresholds in a Cox model controlling for all other potential confounding factors.

\section{Ethical Approval}

The study was approved by the Human Research Ethics Committee of the University of Cape Town (HREC 002/2018).

\section{RESULTS}

Two hundred and eighteen patients diagnosed between 1 January 2000 and 31 December 2015 were included in the analysis with a median follow-up period of 1509 days (IQR 351-2837 days). Patient characteristics are shown in TABLE 1.

\section{Age and Sex}

The majority of patients were females, with a M:F ratio of 1:2.6. Males presented at a median age of 22.5 months (IQR 14.0 - 30.7 months) compared to females at 97 months (IQR 29.0 - 133.7 months). In the univariate analysis, sex was not a significant predictor of survival. However, in the multivariate analysis, females had a significantly lower risk of death (HR $0.284 \mathrm{p}=0.037)$. To test the hypothesis that, among patients with MEGCTs, there existed a potential 'threshold' of age that characterised patients with worse outcomes, the models were refitted modelling age as a penalised-spline. Both the analyses conducted on OS and EFS suggested that, after controlling for all other potential confounding factors, age at diagnosis did not significantly affect the relative risk of death.

\section{Site and histology}

Ovarian masses predominated $(\mathrm{n}=106 ; 48.6 \%)$, reflecting the proportionately larger number of female patients, followed by extragonadal sites (EG) $(n=72 ; 33 \%)$ and lastly testicular masses $(n=40 ; 18.3 \%)$. Yolk sac tumours were most common $(\mathrm{n}=119 ; 54.6 \%)$, followed by dysgerminomas $(\mathrm{n}=53 ; 24.3 \%)$, mixed germ cell tumours $(\mathrm{n}=32 ; 13.8 \%)$, embryonal carcinomas $(\mathrm{n}=8 ; 3.7 \%)$, seminomas $(\mathrm{n}=4 ; 1.8 \%)$ and choriocarcinomas $(\mathrm{n}=2 ; 0.9 \%)$. Neither tumour site nor histology $(\mathrm{p}=0.28)$ was predictive of outcome.

\section{Stage}

Tumours by stage were represented as follows: stage $1(\mathrm{n}=59 ; 27.1 \%)$, stage $2(\mathrm{n}=37 ; 17.0 \%)$, stage $3(\mathrm{n}=75$; $34.4 \%)$ and stage $4(n=46 ; 21.1 \%)$. One patient was unstaged and demised shortly after presentation. Stage was highly predictive of outcome on both univariate $(\mathrm{p}<0.001)$ and multi-variate analysis, especially for patients with stage $3(\mathrm{p}=0.017)$ and stage $4(\mathrm{p}<0.001)$ disease (TABLE 2$)$.

\section{Nutritional Status}

The majority of patients were normally grown $(187 ; 85.8 \%)$ but anthropometric data was missing for four patients. Sixteen patients were moderately stunted whilst 11 were severely stunted. Thirty one were moderately under-weight-for-age (UWFA) and nine were severely UWFA, whilst 12 patients were moderately and severely wasted, respectively. Although the majority of children were not stunted $(87.3 \%)$, wasted $(88.7 \%)$ or UWFA $(81.3 \%)$, both stunting $(\mathrm{p}<0.0001)$ and being UWFA $(\mathrm{p}=0.041)$ appeared to be significant predictors of outcome on univariate analysis but could not be shown to be independently predictive of outcome on multivariate analysis.

Socio-economic Status 
The majority of patients declared their household income per annum (204/218; 93.5\%): 71/204 (34.8\%) patients were fully subsidised, 89/204 (43.6\%) earned a yearly household income of less than USD 6600, 9/204 (4.4\%) earned a yearly household income of between USD 6600 and USD 23000 and 35/204 (17.2\%) earned in excess of USD 23000 per year. Univariate analysis did not demonstrate SES as being a significant determinant of outcome $(\mathrm{p}=0.05)$ but multivariate analysis revealed that patients who came from homes where household income exceeded USD 23000 per year had a significantly lower risk of death (HR 0.071; $\mathrm{p}=0.039$ ) (TABLE 3 ). We also demonstrated a significant negative correlation between SES and stage (Spearman rho $=-0.153$; $\mathrm{p}=0.03$ ), in that patients with higher SES presented with lower stage tumours. However, in the cox model, we adjusted for both SES and stage, revealing that the significant impact of SES on survival was in excess of any effect that could be explained by lower stage disease at diagnosis alone.

\section{Tumour markers}

One hundred and eighty-six patients had secretory tumours with a demonstrably elevated tumour marker at diagnosis: alpha-fetoprotein (AFP) in 157 (81.8\%), beta-human chorionic gonadotropin (b-HCG) in 28 $(14.6 \%)$ and oestradiol in one patient $(0.5 \%)$. Six patients had an elevated lactate dehydrogenase (LDH) in addition to another elevated tumour marker. Using the same methodology for age, we investigated the hypothesis of the existence of a threshold in the relationship between serum AFP levels at diagnosis and outcome. The analysis suggested negligible or no effect of serum AFP levels on OS or EFS for (relatively) small values of AFP, but a rapid increase of the hazard ratio for high values, such that serum AFP levels became significant as a prognostic factor for outcome at a serum AFP level [?] 33,000 ng/ml for OS and a serum AFP level [?] 21,600 ng/ml for EFS ( $\mathrm{p}=0.044)$.

\section{Local Control}

\section{Surgery}

Two hundred and two (93.1\%) patients underwent surgery. One hundred and thirty one had (64.8\%) complete resections whilst $42 / 202(20.7 \%)$ had incomplete resections. Of those who had incomplete resections 12 $(28.6 \%)$ died: nine $(75 \%)$ of disease progression, one from an intra-operative aortic injury and two from other peri-operative complications. Six patients $(6 / 202 ; 2.9 \%)$ had irresectable tumours (two with stage III disease; four with stage IV disease). Four of the six died, three from disease and one from treatment related complications. Ten patients refused surgery: five are dead, all of whom had stage III disease at diagnosis.

\section{Radiotherapy}

Eight patients underwent radiotherapy. Three patients received it as part of upfront therapy to facilitate resection, all with stage III disease. Two are survivors, one is lost to follow-up. Four patients received it as part of relapse therapy. Two are survivors and two are dead. It was used with palliative intent in one patient, who failed chemotherapy and had an irresectable tumour.

\section{Chemotherapy}

Twenty five patients did not receive chemotherapy: 19/25 (76\%) had stage I disease of whom 16 are alive and three are lost to follow-up, presumed alive. Three had stage II disease of whom two died of disease and one is a survivor. Two had stage III disease and one stage IV disease; all of whom died. Chemotherapy was administered to 192 patients: 88 (45.8\%) patients received JEb, 85 (44.2\%) BEP, seven (3.6\%) PEb and 12 $(6.3 \%)$ a variety of non-standard regimens with a mean of 4.7 courses (range 1-11 courses). One hundred medical treatment-related events occurred in 61 patients (Supplemental TABLE S1), the most predictable acute effects being cytopaenias (24) and febrile neutropaenia (11) with hearing loss (19) the commonest subacute/late effect.

\section{Pre-Cisplatin Assessments}

$\mathrm{BEP}$ or PEb was administered to 92 patients. Only nine (9.8\%) had formal two-plasma sample radio-isotope 
GFR assessments whilst calculated GFRs were performed in only six patients. Only 22/92 (23.9\%) patients had formal audiology assessments. In the untested group, unavailability of services was cited for five patients. Hearing loss (ungraded) was reported in 19/22 (86.3\%) patients.

\section{Outcome}

Patients who received chemotherapy had significantly better outcomes compared to those who did not, on both univariate $(\mathrm{p}<0.0007)$ and multivariate $(\mathrm{p}<0.001)$ analysis, irrespective of whether a carboplatin-based or cisplatin-based regimen was used.

In conclusion, only stage $(\mathrm{p}<0.001)$, administration of chemotherapy $(\mathrm{p}<0.001)$ and an elevated serum level of AFP of more than $33,000 \mathrm{ng} / \mathrm{ml}(\mathrm{p}=0.002)$ were independently predictive of OS. The result of the multivariate analysis is summarised in Fig. 1 . The five year OS for the group was acceptable at $80.4 \%$ with an EFS of $75.3 \%$.

\section{DISCUSSION}

This national retrospective study demonstrated that stage, the use of chemotherapy and an elevated serum AFP level at diagnosis were independently predictive of OS. Additionally, female sex was associated with a significantly lower risk of death (HR 0.284; p-value 0.037), even after adjustment for tumour site and histology. The Malignant Germ Cell Tumour International Consortium (MaGIC) Study of 2015 reported that children with yolk sac tumours demonstrated a better outcome, although this was not found to be statistically significant. In addition age, and stage were significant predictors of outcome, but not sex (18). A recent epidemiological study of paediatric germ cell tumours conducted in Danish children revealed a similar female predominance $(71 \%)$ but did not show any relationship between sex and risk of death (25).

In our study tumour stage was significantly predictive of outcome $(\mathrm{p}<0.001)$, especially for patients with advanced disease (Stage III $(\mathrm{p}=0.0171)$ and IV $(\mathrm{p}<0.001))$. Our findings therefore correlated with the MaGIC analysis which demonstrated a statistically significant relationship between stage IV disease, and poor outcome, particularly in children over 11 years of age with advanced stage extragonadal tumours and metastatic ovarian tumours (18). Earlier studies also documented that advanced disease was predictive of poorer outcomes $(26,27)$.

In our cohort a serum AFP level at diagnosis of more than $33,000 \mathrm{ng} / \mathrm{ml}$ was associated with a poorer OS and a level of more than $21,000 \mathrm{ng} / \mathrm{ml}$ was associated with a poorer EFS $(\mathrm{p}=0.044)$. Previously, serum AFP levels of more than 100,000 $\mathrm{g} / \mathrm{L}$ have been associated poorer outcomes in children with hepatoblastoma (28). The MaGIC analysis found that an elevated serum AFP level of more than 10,000 was associated with a poorer outcome but it did not reach the threshold for statistical significance (18). Alpha fetoprotein as a tumour marker has been described as tumour-associated rather than tumour-specific, given that its production can be elevated in a variety of both benign and malignant conditions in children and that the persistence of elevated levels acquired in utero, which usually normalises by 10 months of age ex utero, can sometimes persist until 2 years of age. The elevation of serum AFP levels cannot therefore be considered diagnostic on its own and must be interpreted in concert with other factors (29).

The MaGIC study was able to demonstrate a threshold of age (11 years) beyond which outcomes were poorer using a bootstrap technique (18). We interrogated the same question using age modelled as a penalised spline because of our smaller sample size, and limiting it to patients with more advanced disease, but could not demonstrate a threshold of age beyond which outcomes were either more or less favourable.

In addition to female sex, higher household incomes, in excess of USD 23000 per year, was associated with a significantly lower risk of death (HR0.071; $\mathrm{p}=0.039)$. We also showed a significant relationship between lower stage disease and higher SES. $(\mathrm{p}=0.03)$. However, early presentation alone could not account for this association. This suggests that there are SES factors which may impact outcome during treatment. For example, access to transport may cause delays in chemotherapy or food insecurity may lead to malnutrition, 
negatively impacting immunity and the ability to combat infection or tolerate chemotherapy-related toxicity. Given our past of enforced racial segregation and the association between skin colour and poverty, it is critical to isolate these social determinants of health as skin colour is too easily invoked as a risk factor, when in reality these relate to privilege and access to care. In children with acute lymphoblastic leukaemia a single institutional study reported a higher risk of relapse in children from high poverty areas despite uniform treatment (30). This is relevant given our intention to embark on the implementation of a national treatment protocol for MEGCTs and will be instructive as we interrogate household income and maternal education as measures of SES. Also proving a significant relationship between SES and outcome strengthens our collective call for parity not only in service delivery and access to care but also for adequate social support for children, which is constitutionally enshrined, but not necessarily delivered in real time.

Analysis of our treatment interventions demonstrated that the surgical local control rate in our study was high with a complete resection rate $64.8 \%$. There was a $5 \%$ surgical refusal rate which is a cause for concern considering that half of those patients died of disease as a result. In a systematic review of factors influencing refusal of therapy in adult cancer patients, discomfort of treatment, fear of side effects and transportation difficulties were cited as the most important contributing to refusal of surgery (31). In a recent study from Vietnam adherence to cancer treatment in children was found to be determined by prognosis whilst being associated with local perceptions of cancer and the economic status of affected families (32). It is possible that a more rigorous informed consent and assent process such as that proposed for a national treatment protocol may help to allay the fears associated with paediatric cancer treatment specifically with respect to the surgery which remains a cornerstone of care.

By comparison, the use of chemotherapy was strongly predictive of outcome with no single regimen performing better than any other (carboplatin vs. cisplatin-based) and all regimens producing superior results compared to no chemotherapy at all $(\mathrm{p}<0.001)$. This is corroborated by a recent MaGIC report demonstrating equipoise in contemporaneous trials using cisplatin and carboplatin-based regimens in paediatric germ cell tumours (13).

Radioisotope GFR measurement is recommended as part of the standard of care guidelines for renal function assessment in paediatric oncology practice as it impacts the dosing of platinum alkylators. As such standardising methodologies is critical (33). Rates of formal GFR assessment (even in the absence of radio-isotope instruments) and also audiology assessment were infrequent, which is related to the lack of availability of or access to these services at all POUs across the country, as well as the large burden of patients presenting with advanced disease, whose poor performance status (renal or pulmonary) may preclude radioisotope testing at the time diagnosis. Similarly, the use of calculated GFRs was low in the absence of formal radio-isotope testing. In a more recent study calculated GFRs were found to either over or underestimate the GFR. The recommendation was that radioisotope GFR determination should remain standard of care for platin dosing to ensure accuracy (34). Unfortunately, this may not always be possible in our setting and calculated GFRs may be the best alternative. The value of calculated versus radioisotope GFR measurement can be prospectively tested in the context of a national protocol and calculated GFRs as a surrogate for more accurate renal function evaluation could become standardised practice. In addition, to renal function assessment, significant numbers of hearing-impaired children may have evaded detection because of low rates of routine audiology testing which has the potential to impact school performance and subsequently employability. This is notable because the unemployment rate for the hearing impaired in South Africa ranges from $21.2 \%$ - 35.3\% with low average annual household incomes of approximately USD 2000 for the moderately hearing impaired and USD 1500 for those with severe deficits (35), placing them close to the upper limit poverty line of USD 800 per household per year (36).

Despite these impediments the 5-year OS for the entire cohort was very encouraging $(80.4 \%)$ and patients who relapsed were salvaged (5-year EFS 75.3\%).

We recognise that the sample size and hence the number of events were small and that this could have limited the statistical power to detect significant effects. Different treating centres made use of institutionally specific treatment protocols, often determined by resource constraints. Where space and bed capacity were 
at a premium, outpatient approaches, like JEb was favoured over inpatient regimens like BEP or PEb. Also age cut offs for the admission of adolescents to paediatric wards varied widely between POUs, resulting in an under-representation of older adolescents (16-19 years). Additionally, access to formal radio-isotope testing and audiology testing was fragmented, limiting the accurate assessment of renal function for platin dosing and hearing assessments.

This retrospective analysis was undertaken to assist with the development of a national protocol for children with MEGCTs in South Africa to standardise management and toxicity assessments. In the new national protocol the choice of platinum will be influenced by several factors: firstly, previous local reports of the cost effectiveness of JEb as an outpatient strategy (16) and the significant relationship between SES and outcome reported here; secondly, the rates of platin-related ototoxicity reported here and that under-testing has potentially resulted in a failure to detect significant numbers of hearing impaired children, and; lastly, the availability of audiology services and nuclear medicine facilities which are not ubiquitously available and tend to be clustered near larger POUs in wealthier urban areas.

In addition, the finding of significant relationships between serum AFP levels and SES opens opportunities for the interrogation of these factors in a prospective way in the context of the new protocol as we seek to provide guidance for best practice and as we advocate for social justice in medicine. Creating the discipline of standardised practice by teams with disease-specific expertise able to collaborate across institutional divides will improve paediatric oncology care in South Africa. As we look to the future, we hope to advocate for the establishment of bilateral funding agreements to open doors to children and families from across the sub-continent to receive expert, coordinated care at our national centres of excellence.

\section{Declaration of Conflict of Interest}

The authors have no conflict of interest to declare.

\section{Acknowledgements}

The work has been made possible by support from the National Research Foundation, NRF CSRU180429324830, The Harry Crossley Foundation, University of Cape Town, and The Professor Bongani Mayosi Netcare Clinical Scholarship.

Special thanks to Professor David Reynders and Ms Judy Schoeman of the South African Children's Tumour Registry as well as Mrs Felicity Douglas and Ms Michelle Kannemeyer from the Red Cross War Memorial Children's Hospital in Cape Town. Dr Jaques van Heerden and the staff of the Paediatric Haematology Oncology, Department of Paediatrics and Child Health, Pietermaritzburg Metro Complex, University of Kwa-Zulu-Natal, Pietermaritzburg, South Africa, are also acknowledged for their participation in the study.

\section{Data Availability Statement}

Data restricted due to ethical considerations but reasonable requests will be considered by the corresponding author and only with the express consent of the South African Children's Cancer Study Group (SACCSG).

\section{REFERENCES}

1. Ulbright TM. Germ cell tumors of the gonads: a selective review emphasizing problems in differential diagnosis, newly appreciated, and controversial issues. Mod Pathol. 2005;18 Suppl 2:S61-79.

2. Brodeur GM, Howarth CB, Pratt CB, Caces J, Hustu HO. Malignant germ cell tumors in 57 children and adolescents. Cancer. 1981;48(8):1890-8. 
3. Cancer Incidence and Survival Among Children and Adolescents: Unites States SEER Program 1975-1995. National Cancer Institute, SEER Program Bethesda: NIH Pub; 1999 [No 99-4649:[Available from: http://seer. cancer.gov/publications/childhood.

4. Cushing B, Perlman E, Marina N, Castleberry R. Pinciples and Practice of Pediatric Oncology

. Fifth ed. Philadelphia: Lippincott Williams \& Wilkins 2006.

5. Huang J, Tan Y, Zhen Z, Lu S, Sun F, Zhu J, et al. Role of post-chemotherapy radiation in the management of children and adolescents with primary advanced malignant mediastinal germ cell tumors. PLoS One. 2017;12(8):e0183219.

6. Duchesne GM, Stenning SP, Aass N, Mead GM, Fosså SD, Oliver RT, et al. Radiotherapy after chemotherapy for metastatic seminoma-a diminishing role. MRC Testicular Tumour Working Party. Eur J Cancer. 1997;33(6):829-35.

7. Einhorn LH, Donohue JP. Improved chemotherapy in disseminated testicular cancer. J Urol. 1977;117(1):65-9.

8. Einhorn LH, Donohue JP. Chemotherapy for disseminated testicular cancer. Urol Clin North Am. $1977 ; 4(3): 407-26$.

9. Marina N, Fontanesi J, Kun L, Rao B, Jenkins JJ, Thompson EI, et al. Treatment of childhood germ cell tumors. Review of the St. Jude experience from 1979 to 1988. Cancer. 1992;70(10):2568-75.

10. Mann JR, Raafat F, Robinson K, Imeson J, Gornall P, Phillips M, et al. UKCCSG's germ cell tumour (GCT) studies: improving outcome for children with malignant extracranial non-gonadal tumourscarboplatin, etoposide, and bleomycin are effective and less toxic than previous regimens. United Kingdom Children's Cancer Study Group. Med Pediatr Oncol. 1998;30(4):217-27.

11. Pinkerton CR, Broadbent V, Horwich A, Levitt J, McElwain TJ, Meller ST, et al. 'JEB'-a carboplatin based regimen for malignant germ cell tumours in children. Br J Cancer. 1990;62(2):257-62.

12. Stern JW, Bunin N. Prospective study of carboplatin-based chemotherapy for pediatric germ cell tumors. Med Pediatr Oncol. 2002;39(3):163-7.

13. Frazier AL, Stoneham S, Rodriguez-Galindo C, Dang H, Xia C, Olson TA, et al. Comparison of carboplatin versus cisplatin in the treatment of paediatric extracranial malignant germ cell tumours: A report of the Malignant Germ Cell International Consortium. Eur J Cancer. 2018;98:30-7.

14. Kruger M, Hendricks M, Davidson A, Stefan CD, van Eyssen AL, Uys R, et al. Childhood cancer in Africa. Pediatr Blood Cancer. 2014;61(4):587-92.

15. Stefan DC, Stones DK, Wainwright RD, Kruger M, Davidson A, Poole J, et al. Childhood cancer incidence in South Africa, 1987-2007. S Afr Med J. 2015;105(11):939-47.

16. Hendricks M, Davidson A, Pillay K, Desai F, Millar A. Carboplatin-based chemotherapy and surgery: a cost effective treatment strategy for malignant extracranial germ cell tumours in the developing world. Pediatr Blood Cancer. 2011;57(1):172-4.

17. Marina N, London WB, Frazier AL, Lauer S, Rescorla F, Cushing B, et al. Prognostic factors in children with extragonadal malignant germ cell tumors: a pediatric intergroup study. J Clin Oncol. 2006;24(16):2544-8.

18. Frazier AL, Hale JP, Rodriguez-Galindo C, Dang H, Olson T, Murray MJ, et al. Revised risk classification for pediatric extracranial germ cell tumors based on 25 years of clinical trial data from the United Kingdom and United States. J Clin Oncol. 2015;33(2):195-201.

19. WHO. Nutritional Lanscape Information System (NLIS). Country Profile Indicators Interpretation Guide. Geneva, Switzerland: WHO; 2010. 
20. Kaplan EL, Meier P. Nonparametric Estimation from Incomplete Observations. Journal of the American Statistical Association. 1958;53:457-81.

21. Mantel N. Evaluation of survival data and two new rank order statistics arising in its consideration. Cancer Chemother Rep. 1966;50(3):163-70.

22. Breslow NE. Analysis Of Survival Data Under The Proportional Hazards Model. . International Statistical Review. 1975:45-57.

23. Eilers PHC, Marx BD. Flexible Smoothing With B -Splines And Penalties. Statistical Science. 1996;11:89-121.

24. Grambsch PM, Therneau TM. Proportional Hazards Tests And Diagnostics Based On Weighted Residuals. . Biometrika. 1994;81:515-26.

25. Evers M, Rechnitzer C, Graem N, Skov Wehner P, Schroeder H, Rosthoej S, et al. Epidemiological study of paediatric germ cell tumours revealed the incidence and distribution that was expected, but a low mortality rate. Acta Paediatr. 2017;106(5):779-85.

26. Mann JR, Raafat F, Robinson K, Imeson J, Gornall P, Sokal M, et al. The United Kingdom Children's Cancer Study Group's second germ cell tumor study: carboplatin, etoposide, and bleomycin are effective treatment for children with malignant extracranial germ cell tumors, with acceptable toxicity. J Clin Oncol. 2000;18(22):3809-18.

27. Gobel U, Schneider DT, Calaminus G, Jurgens H, Spaar HJ, Sternschulte W, et al. Multimodal treatment of malignant sacrococcygeal germ cell tumors: a prospective analysis of 66 patients of the German cooperative protocols MAKEI 83/86 and 89. J Clin Oncol. 2001;19(7):1943-50.

28. von Schweinitz D, Bürger D, Bode U, Weinel P, Erttmann R, Hecker H, et al. [Results of the HB-89 Study in treatment of malignant epithelial liver tumors in childhood and concept of a new HB-94 protocol]. Klin Padiatr. 1994;206(4):282-8.

29. Schneider DT, Calaminus G, Göbel U. Diagnostic value of alpha 1-fetoprotein and beta-human chorionic gonadotropin in infancy and childhood. Pediatr Hematol Oncol. 2001;18(1):11-26.

30. Bona K, Blonquist TM, Neuberg DS, Silverman LB, Wolfe J. Impact of Socioeconomic Status on Timing of Relapse and Overall Survival for Children Treated on Dana-Farber Cancer Institute ALL Consortium Protocols (2000-2010). Pediatr Blood Cancer. 2016;63(6):1012-8.

31. Martine TE, Tapscott B, Fitch M, Howell D, Monette J, Wan-Chow-Wah D, et al. A systematic review of factors influencing older adults' decision to accept or decline cancer treatment. Cancer Treatment Reviews. 2015;41(2).

32. Lan BN, Castor A, Wiebe T, Toporski J, Moell C, Hagander L. Adherence to childhood cancer treatment: a prospective cohort study from Northern Vietnam. BMJ Open. 2019;9(8):e026863.

33. Chinnaswamy G, Cole M, Boddy AV, Keir M, Price L, Parry A, et al. Estimation of renal function and its potential impact on carboplatin dosing in children with cancer. Br J Cancer. 2008;99(6):894-9.

34. Shepherd STC, Gillen G, Morrison P, Forte C, MacPherson IR, white JD, et al. Performance of formulae based estimates of glomerularfiltration rate for carboplatin dosing in stage 1 seminoma. Eur $\mathrm{J}$ Cancer. 2014;50(5).

35. [cited 4 February 2020]. Available from: www.stastsa.gov.za/publications/Report-03-01-59/Report03-01-592011.pdf

36. National Poverty Lines. Statistical Release P0310.1 [Internet]. 2018. Available from: http://www . statssa.gov.za/publications/P03101/P031012018.pdf.

\section{LEGENDS}




\section{Legend of Tables}

TABLE 1 Sample Characteristics.

TABLE 2 Five year OS and EFS by Stage.

TABLE 3 The impact of socioeconomic status as a prognostic factor for overall survival.

\section{Legend of Figures}

Figure1 Hazard ratios for overall survival according to multivariate analysis.

\section{Legend of Supplemental Tables}

TABLE S1 Chemotherapy-related Complications.

TABLE 1 Sample Characteristics

\begin{tabular}{|c|c|c|c|}
\hline & Tumour Site & & Total $(\mathrm{n}=218)$ \\
\hline Characteristics & Testicular $(\mathrm{n}=40)$ & Ovarian $(\mathrm{n}=105)$ & $\mathrm{EG}(\mathrm{n}=72)$ \\
\hline \multicolumn{4}{|l|}{ Sex } \\
\hline Male & 40 & 0 & 20 \\
\hline Female & 0 & 106 & 52 \\
\hline \multicolumn{4}{|l|}{ Age } \\
\hline 0 to 4 & 35 & 13 & 59 \\
\hline 5 to 9 & 2 & 29 & 6 \\
\hline 10 to 14 & 2 & 58 & 6 \\
\hline${ }^{3} 15$ & 1 & 6 & 1 \\
\hline \multicolumn{4}{|l|}{ Tumour site } \\
\hline Ovarian & 0 & 106 & 0 \\
\hline Testicular & 40 & 0 & 0 \\
\hline Retroperitoneal & 0 & 0 & 30 \\
\hline Abdomen & 0 & 0 & 13 \\
\hline Mediastinal & 0 & 0 & 13 \\
\hline Sacrococcygeal & 0 & 0 & 11 \\
\hline Head \& Neck & 0 & 0 & 5 \\
\hline \multicolumn{4}{|l|}{ Histology } \\
\hline Yolk sac tumour & 31 & 37 & 51 \\
\hline Mixed germ cell tumour & 3 & 14 & 15 \\
\hline Embryonal carcinoma & 3 & 5 & 0 \\
\hline Choriocarcinoma & 0 & 1 & 1 \\
\hline Dysgerminoma & 0 & 49 & 4 \\
\hline Seminoma & 3 & 0 & 1 \\
\hline \multicolumn{4}{|l|}{ Stage } \\
\hline I & 19 & 27 & 13 \\
\hline II & 5 & 22 & 10 \\
\hline III & 9 & 39 & 27 \\
\hline IV & 7 & 18 & 21 \\
\hline Missing & 0 & 0 & 1 \\
\hline \multicolumn{4}{|l|}{ Stunted } \\
\hline None & 35 & 88 & 64 \\
\hline Moderate & 1 & 12 & 3 \\
\hline
\end{tabular}




\begin{tabular}{llll} 
Severe & 2 & 4 & 5 \\
Missing & 2 & 2 & 0 \\
Wasted & & & \\
None & 38 & 91 & 61 \\
Moderate & 0 & 6 & 6 \\
Severe & 0 & 7 & 5 \\
Missing & 2 & 2 & 0 \\
UWFA & & & \\
None & 35 & 82 & 58 \\
Moderate & 4 & 17 & 10 \\
Severe & 0 & 5 & 0 \\
Missing & 1 & 2 & 19 \\
AFP [ng/mL] & & & 42 \\
0 to 9999 & 19 & 22 & 11 \\
3 10000 & 12 & 36 & 4 \\
Missing & 9 & 48 & 37 \\
Treatment Regimen & & & 4 \\
No chemotherapy & 15 & 7 & 22 \\
JEb & 13 & 38 & 5 \\
PEb & 1 & 2 & 53 \\
BEP & 10 & 1 & \\
Other & 1 & & \\
\hline
\end{tabular}

EG - extragonadal, AFP - alpha fetoprotein, UWFA - under-weight-for-age, JEb - carboplatin, etoposide, bleomycin; PEb - cisplatin, etoposide, bleomycin; BEP - bleomycin, etoposide, cisplatin.

TABLE 2 Five year OS and EFS by Stage.

\begin{tabular}{lllll}
\hline Stage & OS $(\%)$ & EFS $(\%)$ & $\begin{array}{l}\text { Test for differences } \\
\text { in survival } \\
\text { distributions } \\
(\mathrm{p} \text {-value) }\end{array}$ & $\begin{array}{l}\text { Cox Regression } \\
\text { (Multivariate } \\
\text { analysis) }\end{array}$ \\
I & 96.0 & OS & EFS \\
II & 94.3 & 94.1 & $\begin{array}{l}\mathrm{p}<0.001 \\
\mathrm{p}=0.7195\end{array}$ & $\mathrm{p}<0.001$ \\
III & 75.5 & 85 & $\begin{array}{l}\mathrm{p}=0.0171 \\
\mathrm{p}<0.001\end{array}$ & \\
IV & 60.1 & 69.8 & & \\
\hline
\end{tabular}

OS Overall survival, EFS Event free survival

TABLE 3 The impact of socioeconomic status as a prognostic factor for overall survival.

Household Income/Year

n $(\%)$

OS 
Fully subsidised

$<$ USD 6600

USD 6600-23100

> USD 23100
$71(34.8 \%)$

$89(43.6 \%)$

$9(4.4 \%)$

$35(17.2 \%)$
Univariate Analysis

$\mathrm{p}=0.05$

$\mathrm{p}=0.6835$

$\mathrm{p}=0.8992$

$\mathrm{p}=0.039$ 